\title{
Recomendações para a utilização de oxigênio suplementar (oxigenoterapia) em pacientes com COVID-19*
}

\author{
Recommendations for the use of supplemental oxygen therapy in patients with COVID-19*
}

\author{
SILVA, Vinícius Zacarias Maldaner da ${ }^{1}$; NEVES, Laura Maria Tomazi²; FORGIARINI Jr, \\ Luiz Alberto ${ }^{3}$; em nome do Comitê COVID-19 da ASSOBRAFIR.
}

\begin{abstract}
Resumo
Este documento procura esclarecer quanto à utilização de oxigênio suplementar (oxigenoterapia), e suas formas de administração, para o tratamento da insuficiência respiratória aguda causada pela COVID-19. Recomenda-se o início da oxigenioterapia com fluxo de $5 \mathrm{~L} / \mathrm{min}$ com alvo de $\mathrm{SpO}_{2} \geq 94 \%$ para pacientes com algum sinal de instabilidade clínica e alvo de $\mathrm{SpO}_{2} \geq 90 \%$ quando o paciente estiver estável ou $\mathrm{SpO}_{2} \geq$ 92\% para gestantes. Recomenda-se a utilização de cateter nasal de oxigênio com até 5L/min sem necessidade de umidificação e não se recomenda a utilização de máscaras de Venturi®. Se o paciente não atingir a $\mathrm{SpO}_{2}$ alvo, recomenda-se ajustar o fluxo de $\mathrm{O}_{2}$ entre 10 e $15 \mathrm{~L} / \mathrm{min}$ com máscara reservatório não reinalante. $\mathrm{O}$ posicionamento da ASSOBRAFIR visa que o Fisioterapeuta adote estratégias de oxigenoterapia adequadas, auxiliando na sobrevida e demais desfechos negativos dos pacientes com COVID-19 e na prevenção das disfunções respiratórias e funcionais características desta infecção.
\end{abstract}

Palavras-chave: Fisioterapia; Oxigenoterapia; COVID-19.

\footnotetext{
* Revisado por membros do Comitê COVID-19 da ASSOBRAFIR, nomeado por meio do memorando Nº 003/2020. Esta publicação é uma atualização da Comunicação Oficial "Recomendações para a utilização de oxigênio suplementar (oxigenoterapia) em pacientes com COVID-19", chancelada pelo Comitê COVID-19 da ASSOBRAFIR, originalmente escrita pelos mesmos autores e divulgada em 20/06/2020 no endereço eletrônico https://assobrafir.com.br/covid-19oxigenoterapia/

${ }^{1}$ Escola Superior de Ciências da Saúde (ESCS), Secretaria de Saúde do Distrito Federal (SES-DF). Email: viniciusmaldaner@gmail.com. VZMS - http://orcid.org/0000-0002-7804-7517

${ }^{2}$ Laboratório de Avaliação e Reabilitação das Disfunções Cardiovascular, Oncológica e Respiratória (LACOR), Programa de Pós-Graduação em Ciências do Movimento Humano (PGCMH), Instituto de Ciências da Saúde (ICS), Universidade Federal do Pará (UFPA). Complexo Hospitalar da Universidade Federal do Pará (CH-UFPA).

LMTN - https://orcid.org/0000-0002-3115-2571

${ }^{3}$ Docente do Curso de Fisioterapia e Programa de Pós-graduação em Saúde e Desenvolvimento Humano na Universidade La Salle. LAF - http://orcid.org/0000-0002-6706-2703
} 


\section{Abstract}

This document seeks to clarify the use of supplemental oxygen (oxygen therapy) for the treatment of acute respiratory failure caused by COVID-19. It is recommended to start oxygen therapy with flow rate of 5L/min with the $\mathrm{SpO}_{2}$ target $\geq 94 \%$ for patients with signs of clinical instability and the $\mathrm{SpO}_{2}$ target $\geq 90 \%$ when the patient is stable or $\mathrm{SpO}_{2} \geq 92 \%$ for pregnant women. It is recommended to use nasal oxygen catheter with up to $5 \mathrm{~L} / \mathrm{min}$ without humidification. The use of Venturi ${ }^{\circledR}$ masks is not recommended. If the patient does not reach the target $\mathrm{SpO}_{2}$, it is recommended to adjust the $\mathrm{O}_{2}$ flow between 10 and $15 \mathrm{~L} / \mathrm{min}$ with a non-rebreather mask. The ASSOBRAFIR positioning aims that the Physiotherapist adopts appropriate oxygen therapy strategies, aiding in the survival and other negative outcomes of patients with COVID-19 and in preventing the respiratory and functional dysfunctions characteristic of this infection.

Keywords: Physiotherapy; Oxygen Inhalation Therapy; COVID-19.

\section{Objetivo}

Este documento tem como objetivo esclarecer quanto à utilização de oxigênio suplementar (oxigenoterapia), e suas formas de administração, para o tratamento da insuficiência respiratória aguda (IRpA) causada pela COVID-19. Nesse contexto, a segurança do paciente e dos profissionais de saúde envolvidos na linha de frente de cuidado são aspectos relevantes devido ao risco de geração de aerossóis e a propagação do vírus pelo ar.

\section{Contextualização}

A manutenção da oferta de oxigênio às células, atendendo à demanda metabólica é uma função crucial do sistema cardiorrespiratório. Sob condições normais, a oferta de oxigênio às células é controlada por um processo denominado "oferta direcionada pela demanda" . Em várias doenças a manipulação das variáveis que interferem no transporte sistêmico de oxigênio $\left(\mathrm{TO}_{2}\right)$, visa adequar a oferta à demanda metabólica. Pode-se dizer que o $\mathrm{TO}_{2}$ se inicia quando o oxigênio é captado da atmosfera, difundindo-se do espaço alveolar para o sangue capilar, onde é transportado ligado à hemoglobina (caracterizando a saturação arterial de oxigênio da hemoglobina $-\mathrm{SaO}_{2}$ ) e, em pequena parte, dissolvido no plasma (caracterizando a pressão parcial de oxigênio no plasma arterial $\left.-\mathrm{PaO}_{2}\right)^{2}$. Então, a partir da contração cardíaca, o fluxo sanguíneo é distribuído na circulação sistêmica e, posteriormente, na microcirculação. Finalmente, o oxigênio é liberado da hemoglobina e se difunde do espaço funções vitais em nível celular, o que se traduz na obtenção de energia. $\mathrm{O}$ perfeito entendimento dos processos fisiopatológicos, que podem aí interagir, é fundamental para a correta interpretação clínica e laboratorial das manifestações apresentadas pelo paciente, com consequente adequada intervenção terapêutica. Essa seria uma maneira simplista de descrevermos o $\mathrm{TO}_{2}$, sendo que o mesmo é o produto do débito cardíaco (DC) e do conteúdo arterial de $\mathrm{O}_{2}\left(\mathrm{CaO}_{2}\right)$ como denotado na equação de Fick: $\mathrm{TO}_{2}=\mathrm{CaO}_{2}$ x DC x k, onde $\mathrm{CaO}_{2}=\left(\mathrm{Hb} \times \mathrm{SaO}_{2} \times 1,34\right)+(0,003$ $\mathrm{x} \mathrm{PaO}_{2}$ ); $\mathrm{k}$ - constante; $\mathrm{Hb}$ - hemoglobina; $\mathrm{PaO}_{2}$ - pressão parcial de oxigênio; 1,34 - quantidade de $\mathrm{O}_{2}$ que $1,0 \mathrm{~g}$ de $\mathrm{Hb}$ consegue transportar. Embora $\mathrm{o} \mathrm{TO}_{2}$ seja manipulado na tentativa de adequar $\mathrm{o}$ desequilíbrio entre oferta e consumo de $\mathrm{O}_{2}$, o que, de fato, chega à célula é denominado oferta de $\mathrm{O}_{2}$ $\left(\mathrm{DO}_{2}\right)$.

Em relação ao $\mathrm{TO}_{2}$, conseguimos avaliá-lo por meio de dois exames: a) gasometria arterial, no qual obtemos a saturação arterial de oxigênio $\left(\mathrm{SaO}_{2}\right)$, que em indivíduos saudáveis varia de 96 a 
98\%; e b) oximetria de pulso, método não invasivo que por meio de um feixe de luz consegue medir a saturação periférica de oxigênio $\left(\mathrm{SpO}_{2}\right)$, a qual geralmente tem uma variação de 2-3\% em relação à $\mathrm{SaO}_{2}{ }^{3}$.

A oxigenoterapia consiste na administração de oxigênio acima da concentração do ar ambiente $(\sim 21 \%)$ e tem por objetivo garantir a oxigenação dos tecidos. Ela é utilizada para corrigir hipoxemia e, consequentemente, promover a diminuição da sobrecarga de trabalho cardiorrespiratório, mediante a elevação dos níveis alveolar e sanguíneo de oxigênio ${ }^{4}$. A causa mais frequente para a utilização da oxigenoterapia é a insuficiência respiratória aguda (IRpA), em que há impossibilidade do sistema respiratório manter os valores da pressão arterial de oxigênio $\left(\mathrm{PaO}_{2}\right)$ e/ou da pressão arterial de gás carbônico $\left(\mathrm{PaCO}_{2}\right)$. Para a American Association for Respiratory Care, a oxigenoterapia é inidicada, principalmente, na presença de $\mathrm{PaO}_{2}<60 \mathrm{mmHg}$ ou $\mathrm{SpO}_{2}<90 \%$, em ar ambiente, e/ou $\mathrm{SpO}_{2}<88 \%$ durante o exercício ou sono, em pacientes com doenças cardiorrespiratórias. ${ }^{5}$

Como não há estudos randomizados ou não randomizados sobre o uso de oxigenoterapia em adultos com COVID-19, utilizamos evidências descritas em outras populações ou doenças para elaborar o presente posicionamento. Uma revisão sistemática com meta-análise de 25 ensaios clínicos randomizados (16.037 pacientes) demonstrou que uma estratégia liberal de oxigênio sem uma $\mathrm{SpO}_{2}$ mínima alvo está associada ao aumento do risco de mortalidade hospitalar (RR 1,21 IC 95\% = 1,03 - 1,43) em pacientes críticos sem COVID- $19^{3}$ e um recente guia de recomendações práticas afirma que a $\mathrm{SpO}_{2}$ deve ser mantida entre 90 e 96\%, não havendo necessidade de suplementação para valores acima de $96 \%{ }^{4}$.

No contexto da COVID-19, um estudo chinês com 1009 pacientes mostrou que $41 \%$ de todos os doentes foram hospitalizados e mais de $70 \%$ daqueles com a forma grave da doença necessitaram de oxigênio suplementar ${ }^{1}$. Para os pacientes críticos a hipóxia pode ser prejudicial e está associada a piores resultados, ou seja, aumento da morbidade 2 .

Considerando os prejuízos da hiperóxia e de valores elevados de $\mathrm{SpO}_{2}$ (próximas a 98-100\%), o aumento de custo em relação a maior demanda de oxigenioterapia pelos pacientes com COVID-19, e o aumento da mortalidade nessa população com hipoxemia, recomenda-se manter, durante oxigenioterapia, $\mathrm{SpO}_{2}$ entre 92 e $96 \%$.

\section{Oxigenoterapia e a COVID-19}

Em relação à pandemia da COVID-19, a doença pode apresentar 3 grandes fenótipos relacionados ao comprotimento pulmonar ${ }^{6}$ :

- Fenótipo 1, com opacidades múltiplas em vidro fosco, focais e possivelmente com baixa perfusão;

- Fenótipo 2, com atelectasias com distribuição heterogênea e opacidades peribrônquicas;

- Fenótipo 3, padrão compatível com a síndrome do desconforto respiratório agudo (SDRA).

Alguns pacientes apresentam hipoxemia sem outros sinais de insuficiência respiratória, como tiragens, aumento da frequência respiratória e uso de musculatura acessória da respiração. Essa situação é denominada de happy hypoxemia ou silent hypoxemia ${ }^{7}$. Essas características apresentadas levam a um desequilíbrio da relação ventilação/perfusão e, consequentemente, à hipoxemia ${ }^{4}$.

Para iniciar a oxigenoterapia, podemos seguir o fluxograma da Figura 1. Segundo a Organização 
Mundial de Saúde $(\mathrm{OMS})^{5}$, indica-se o início da oxigenioterapia com fluxo de $5 \mathrm{~L} / \mathrm{min}$ com o alvo de $\mathrm{SpO}_{2} \geq 94 \%$ para pacientes com algum sinal de instabilidade clínica, como insuficiência respiratória, hipoxemia ou choque; e alvo de $\underline{\mathrm{SpO}}_{2} \geq 90 \%$ quando o paciente estiver estável ou $\underline{\mathrm{SpO}}_{2} \geq 92 \%$ para gestantes. Considerando-se o nível de evidência, recomenda-se início da oxigenoterapia quando a $\mathrm{SpO}_{2}$ for menor que $90 \%$ (forte recomendação com moderada evidência) e sugere-se iniciar oxigenoterapia caso a SpO2 esteja abaixo de 92\% (recomendação fraca, com baixo nível de evidência). Em ambas as situações, recomendada-se $\mathrm{SpO}_{2}$ alvo de até $94 \%$ (forte recomendação com moderada evidência $)^{6}$. Em relação a interface, não recomenda-se a utilização de máscaras de Venturiß para oxigenoterapia a esses pacientes (moderada recomendação com moderada evidência).

Recomenda-se a utilização de cateter nasal de oxigênio com até $5 \mathrm{~L} / \mathrm{min}$ sem necessidade de umidificação para reduzir produção de aerossóis e o risco de infecção por outros patógenos (forte recomendação com moderada evidência) para início da terapia. Pode-se utilizar a máscara cirúrgica sobre o dispositivo (fraca recomendação, com fraca evidência). Se o paciente não atingir a $\underline{S p O}_{2} \underline{a l v o}$, recomenda-se ajustar o fluxo de $\mathrm{O}_{2}$ entre 10 e $15 \mathrm{~L} / \mathrm{min}$ com máscara reservatório não reinalante.

Figura 1 | Fluxograma para oxigenioterapia nos pacientes com COVID-19.

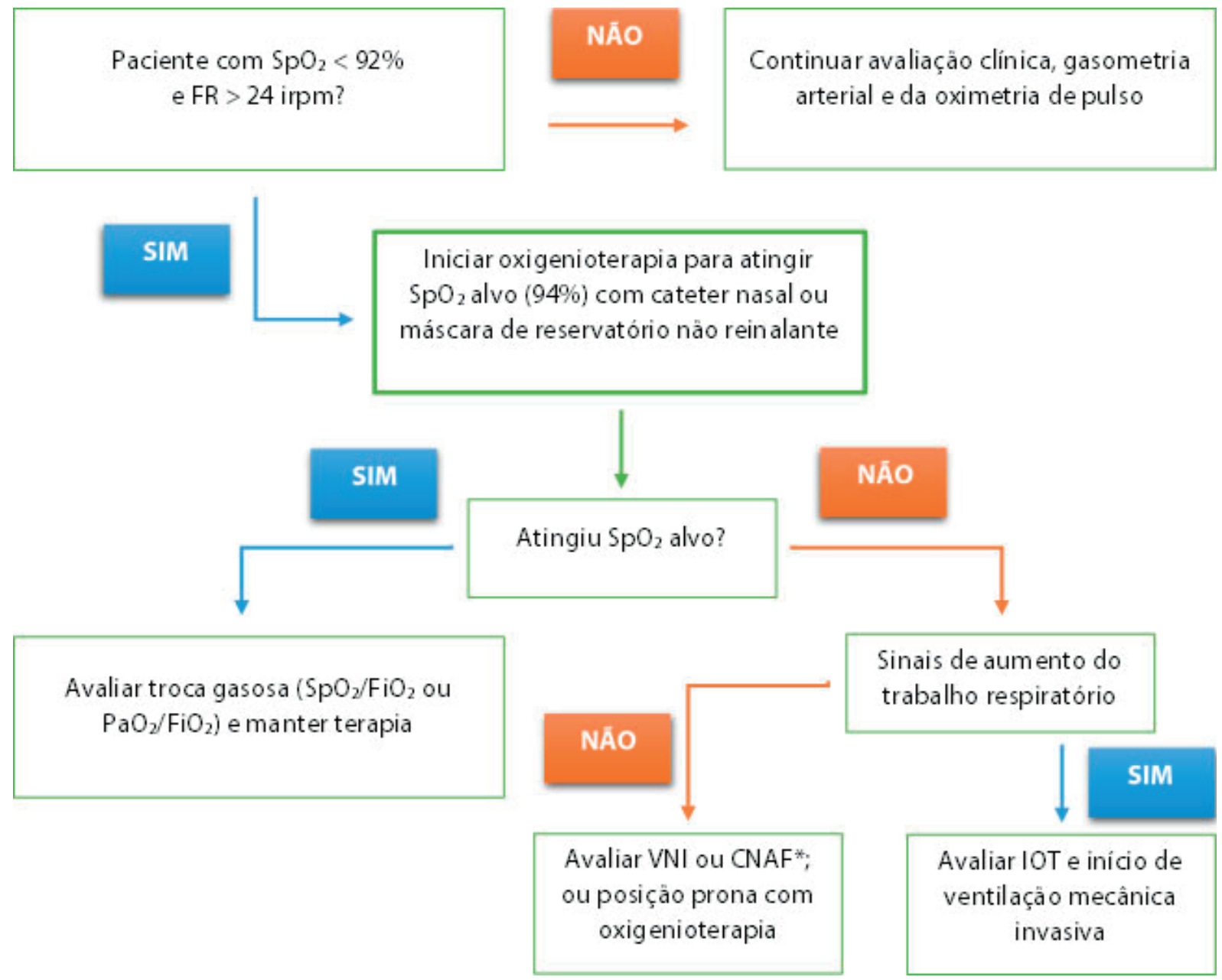

Legenda: $\mathrm{SpO}_{2}$ - saturação periférica de oxigênio; $\mathrm{FR}$ - frequência respiratória; $\mathrm{FiO}_{2}$ - fração inspirada de oxigênio; $\mathrm{PaO}_{2}$ - pressão arterial de oxigênio; $\mathrm{VNI}$ - ventilação não invasiva; CNAF - cânula nasal de alto fluxo; IOT - intubação orotraqueal; *sob condições específicas.

Fonte: elaborada pelos autores e Comitê COVID-19. 


\section{Considerações Finais}

É crescente no Brasil o número de pacientes com COVID-19 que evoluem com IRpA e precisam ser hospitalizados para correção da hipoxemia. É fundamental que os Fisioterapeutas estejam capacitados para gerenciar de maneira eficiente a oferta de oxigênio aos pacientes e também saber que existem riscos, de modo a evitar a disseminação do vírus, por meio da utilização de recursos adequados e com o emprego dos EPIs, conforme preconizados pela Organização Mundial de Saúde e pela ASSOBRAFIR. Também é fundamental conhecer quais formas de administração devem ser utilizadas e quais parâmetros devem ser avaliados durante a oxigenoterapia. O posicionamento da ASSOBRAFIR visa que o Fisioterapeuta que atua na linha de frente auxilie a garantir a sobrevida dos pacientes com COVID-19, adotando estratégias de oxigenoterapia adequadas, reduzindo os desfechos negativos tão frequentes nesta doença. Além disso, visa que o Fisioterapeuta previna as disfunções respiratórias e funcionais características desta infecção.

Este é o posicionamento da ASSOBRAFIR em relação às recomendações para a utilização de oxigenoterapia em pacientes com COVID-19. Esperamos, com isso, contribuir para a orientação e esclarecimento dos fisioterapeutas neste momento de incertezas. A ASSOBRAFIR está atenta à evolução dos acontecimentos e sempre que identificar necessidade emitirá nova comunicação.

\section{Referências}

1. Siemieniuk RAC, Chu DK, Kim LH, Guell-Rous MR, Alhazzani W, Soccal PM, et al. Oxygen therapy for acutely ill medical patients: a clinical practice guideline. BMJ. 2018 Oct 24;363:k4169. doi: 10.1136/bmj.k4169.

2. Comité Nacional de Neumonología. [Guidelines for home oxygen therapy management: Part 1 : generality, indications and monitoring]. Arch Argent Pediatr. 2013 Oct;111(5):448-54. doi: 10.5546/ aap.2013.448. Spanish

3. Magnet FS, Schwarz SB, Callegari J, Criee CP, Storre JH, Windisch W. Long-Term Oxygen Therapy: Comparison of the German and British Guidelines. Respiration. 2017;93(4):253-263. doi: 10.1159/000455879. Epub 2017 Feb 1.

4. Thomas P, Baldwin C, Bissett B, Boden I, Gosselink R, Granger CL, et al. Physiotherapy management for COVID-19 in the acute hospital setting: clinical practice recommendations. J Physiother. 2020 Apr;66(2):73-82. doi: 10.1016/j.jphys.2020.03.011. Epub 2020 Mar 30.

5. AARC clinical practice guideline. Oxygen therapy in the home or alternate site health care facility--2007 revision \& update. Respir Care. 2007 Aug;52(8):1063-8.

6. Rello J, Storti E, Belliato M, Serrano R. Clinical phenotypes of SARS-CoV-2: Implications for clinicians and researchers. Eur Respir J. 2020 May; 55(5): 2001028. Published online 2020 May 21. doi: 10.1183/13993003.01028-2020.

7. Ottestad W, Seim M, Maehlen JO. COVID-19 with silent hypoxemia. Tidsskr Nor Laegeforen. 2020 Apr 11;140(7). doi: 10.4045/tidsskr.20.0299.

Submissão em: 05/06/2020

Aceite em: 15/07/2020 